\title{
La (ri)scoperta di Dante in Francia tra secolo dei Lumi e primo Ottocento
}

\section{Franco Piva}

\section{(2) OpenEdition}

1 Journals

\section{Edizione digitale}

URL: http://journals.openedition.org/studifrancesi/7791

DOI: ERREUR PDO dans/localdata/www-bin/Core/Core/Db/Db.class.php L.34 : SQLSTATE[HYO0O]

[2006] MySQL server has gone away

ISSN: 2421-5856

\section{Editore}

Rosenberg \& Sellier

\section{Edizione cartacea}

Data di pubblicazione: 1 juillet 2009

Paginazione: $264-277$

ISSN: 0039-2944

\section{Notizia bibliografica digitale}

Franco Piva, «La (ri)scoperta di Dante in Francia tra secolo dei Lumi e primo Ottocento», Studi Francesi [Online], 158 (LIII | II) | 2009, online dal 30 novembre 2015, consultato il 07 janvier 2021. URL: http:// journals.openedition.org/studifrancesi/7791 ; DOI: https://doi.org/ERREUR PDO dans /localdata/ www-bin/Core/Core/Db/Db.class.php L.34 : SQLSTATE[HY000] [2006] MySQL server has gone away

\section{(c) (†) $\odot$}

Studi Francesi è distribuita con Licenza Creative Commons Attribuzione - Non commerciale - Non opere derivate 4.0 Internazionale. 


\section{La (ri)scoperta di Dante in Francia tra secolo dei Lumi e primo Ottocento}

Una profonda conoscitrice di Dante e della sua fortuna in Francia come Jacqueline Risset, dopo aver fatto notare, nel corso di un convegno svoltosi alcuni anni orsono, che «La France est probablement le seul pays européen à ne pas avoir intégré l'œuvre de Dante dans l'histoire de sa littérature», ha affermato che la storia del rapporto che la Francia ha intrattenuto attraverso i secoli con Dante è, di fatto, la storia di un'assenza ${ }^{1}$; o, se si preferisce, e per essere più precisi, quella di un rapporto sostanzialmente, e per lungo tempo, mancato, nonostante alcuni indizi di un interesse più o meno intenso, che tuttavia non è mai riuscito a trasformarsi in presenza definitiva, e un momento, quello romantico, in cui il rapporto della Francia con Dante e la sua opera assunse, o sembrò assumere la dimensione di quello che Jacqueline Risset ha chiamato un inatteso, quanto improvviso, «engouement collectif»?2. La sostanziale esattezza di questa osservazione, almeno per quanto riguarda il periodo da noi preso in considerazione, sta nei fatti, che la stessa Risset in quel suo intervento ha evocato brevemente, e che altri, prima e dopo di lei, avevano ed hanno avuto modo di ricordare in maniera più articolata ${ }^{3}$. La prima traduzione della Divina Commedia uscì in Francia solo alla fine del XVI secolo. Dedicata ad Enrico IV, la traduzione di Balthazard Grangier ${ }^{4}$ non sembra però avere incontrato un grande successo di pubblico; né successo maggiore conobbero altri tentativi, parziali o totali, intrapresi prima e dopo quello di Grangier ${ }^{5}$; rimasti per lo più manoscritti, essi ebbero per forza

(1) J. Risset, Dante en France. Histoire d'une absence, in L'Italia letteraria e l'Europa. Atti del Convegno di Aosta 20-23 ottobre 1997, Roma, Sellerio, 2001, p. 59.

(2) Ibid. J. Risset non è stata, peraltro, la prima ad affermare questo, che costituisce anzi una sorta di luogo comune nella critica d'oltralpe. Albert Counson, il primo serio studioso della fortuna di Dante in Francia, aveva fatto notare fin dal 1905 che «Dante n'avait rencontré chez les écrivains français que l'oubli, l'indifférence et le dédain, quand le romantisme vint lui faire une gloire soudaine et bruyante» (Dante et les romantiques français, «Revue d'Histoire Littéraire de la France», 12ème année (1905), p. 361). In anni più recenti, André PÉZARD, un altro profondo conoscitore di Dante in Francia, ha ribadito che «Dante, mal connu, mal jugé des Français au XVII et au XVIII ${ }^{e}$ siècle, devient tout d'un coup merveilleusement populaire vers 1830» (Comment Dante conquit la France aux beaux jours du Romantisme, in Studi in onore di Carlo Pellegrini, Torino, SEI, 1963, p. 683).

(3) In particolare Albert Counson (Dante en France, Erlangen, Fr. Junge - Paris, Fontmoing, 1906), riprendendo e completando lo studio indicato alla nota precedente, e Michael PITWOOD
(Dante and the French Romanticism, Genève, Droz 1985), indubbiamente i due studiosi più accreditati della fortuna di Dante in Francia.

(4) Cfr. La Comédie de Dante, de l'Enfer, du Purgatore et du Paradis, mise en ryme françoise \& commentée par M. Balthazar GrangIER, Conseiller \& Aulmonier du Roy \& Abbé de S. Barthelemi de Noyon. A Paris, chez la Veuve Drobet, 1596. La traduzione (ci informa Marc SCIALOM, Répertoire chronologique et raisonné des traductions françaises de la Divine Comédie ( $X V^{\circ}-X X^{e}$ siècles), «Lingua e Letteratura», 7 [1986], p. 124), «en stophes de six alexandrins rimés selon le schéma ABABCC», comportava una «Epître dédicatoire à Henri IV», delle note «empruntées à G. Landino et A. Vellutello»e un «Répertoire des plus signalées matieres contenues ès trois Cantiques de la Comédie de Dante».

(5) Ricordiamo la traduzione, parziale, del Paradiso, ad opera di François Bergaigne, della prima metà del XVI secolo, conservata alla Bibliothèque Nationale di Parigi, quella, anonima, della Divina Commedia, conservata manoscritta alla Biblioteca Nazionale di Vienna, della seconda metà del secolo, e la traduzione dell'Inferno, ad opera di Philippe Le Hardy, della prima metà del XVII secolo, conservata alla Biblioteca Municipale di Tolosa. 
di cose una circolazione, e quindi un'incidenza sulla diffusione di Dante in Francia, assai limitate. Altrettanto scarsa fu l'accoglienza che a Dante riservarono i letterati d'Oltralpe: se si eccettuano Christine de Pizan, che, agli inizi del Quattrocento, osò porre la Commedia al di sopra del Roman de la Rose, e Marguerite de Navarre che, un secolo più tardi, creò, con il fratello, il futuro Francesco I, una sorta di Accademia dantesca, e che a Dante, specie nell'ultima parte della sua vita, quella più provata dal dolore, portò un vero e proprio culto ${ }^{6}$, gli uomini di lettere francesi che si avvicinarono seriamente a Dante o che della sua poesia subirono l'influenza furono pochi: Alain Chartier, Martin Lefranc, Maurice Scève, sul quale si è soffermata in particolar modo J. Risset, e pochi altri. A Dante i Francesi preferirono presto il più elegante Petrarca ed il petrarchismo.

La situazione divenne ancora più netta con il Rinascimento ed il progressivo imporsi del Classicismo. Una delle ragioni dello scarso successo e della scarsa influenza della traduzione del Grangier sta proprio nel fatto che essa si situa al limite estremo del pur modesto interesse che i Francesi avevano riservato a Dante. La Commedia fu relegata tra le cose vecchie e di cattivo gusto. I pochi che ne parlarono, Chapelain, Rapin o Boileau, lo fecero per condannare la mancanza di ogni più elementare regola del bello scrivere che, a loro avviso almeno, il poema dantesco inesorabilmente rivelava ${ }^{7}$.

Erede del Classicismo seicentesco e del gusto che esso aveva prodotto in letteratura, il Settecento non poteva, di per sé, apprezzare Dante, e tanto meno la sua Commedia, così lontana da quell'ordine e da quelle regole, del cui rispetto il secolo dei Lumi aveva fatto una sorta di dogma. Agli occhi di Voltaire e di Laharpe il capolavoro di Dante non era che una «production bizarre» quando non addirittura un «monstre» che il raffinato gusto settecentesco non poteva assolutamente accettare ${ }^{8}$. Fu tuttavia proprio nel Settecento che la riscoperta di Dante prese avvio. Non furono pochi in quel secolo coloro che, in modo più o meno cosciente, si resero conto che il Classicismo, ormai esausto, aveva bisogno di nuova linfa, di nuova energia, di nuova ispirazione; un'energia ed una ispirazione che alcuni credettero di poter trovare nelle letterature straniere, in quella inglese, certo, con Ossian e soprattutto con Shakespeare, ma anche in quella italiana. Se, agli occhi della maggior parte dei letterati francesi, Dante era, come Shakespeare, un barbaro ignorante, la sua poesia possedeva però una forza ed un fascino ai quali certuni non furono affatto insensibili. Ad esempio Charles de Brosses, il futuro Président à mortier del Parlement de Bourgogne, che scoprì Dante durante il suo viaggio in Italia, quindi negli anni 1739-1740. Se la sua cultura classica gli fece trovare inizialmente Dante «difficile à entendre, tant par son style que par ses allégories, car un sublime dur se trouve enveloppé dans un langage obscur», in quel «langage obscur» il raffinato viaggiatore francese sentì tuttavia una forza poetica ed una grandezza da cui si lasciò ben presto affascinare, sicché l'iniziale avversione finì col trasformarsi in un'insopprimibile ammirazione: «Je l'admire - confessò infatti ad un amico - comme un rare génie, surtout pour le temps où il a vécu (sur la fin du XIII ${ }^{\mathrm{e}}$ siècle) et comme le premier homme de l'Europe qui, dans les siècles modernes, ait vraiment mérité le nom de poète»`. Ciò che ini-

(6) A questo proposito, oltre a quanto detto da J. Risset nell'intervento sopra citato, cfr. A.Counson, Dante en France cit., pp. 7-27 e M. PITWOOD, op. cit., pp. 22-27.

(7) Cfr. quanto ha scritto, a questo proposito, A. Counson, Dante en France, cit., pp. 52-58.

(8) Per l'atteggiamento assunto, in particolare, da Voltaire nei riguardi di Dante cfr., tra gli altri,
A. Farinelli, Dante e la Francia, dall'età media al secolo di Voltaire, Milano, Hoepli, 1908, t. II, cap. «Voltaire e il suo secolo».

(9) Ch. DE Brosses, Lettres familières. Texte établi par Giuseppina CAFASSO. Introduction, notes et bibliographie par Letizia NorCI CAGIANO DE AzEvEDO, Napoli, Centre Jean Bérard, 1991, pp. 890-891 (Lettre XLII a Monsieur de Neuilly). 
zialmente aveva tenuto il futuro Président de Brosses lontano da Dante era stata, tra l'altro, la profonda tristezza che emanava da quella poesia ${ }^{10}$ : ma una ventina di anni più tardi fu proprio quella tristezza che attrasse a Dante un altro giovane francese, Baculard d'Arnaud che nell'Inferno del poeta italiano trovò uno dei più significativi esempi di quel «sombre» che per lo scrittore d'Oltralpe era «peut-être la première magie du pittoresque», e sul quale egli fondò il suo ambizioso progetto di rinnovare la poesia, soprattutto teatrale, del suo tempo ${ }^{11}$. Del resto se Voltaire non aveva visto inizialmente nella Commedia che «un poème bizarre», nel 1756 egli, con più calma e onestà, riconobbe che il poema dantesco era un «ouvrage dans lequel l'auteur [s'était élevé] au-dessus du mauvais goût de son siècle et de son sujet, et [che era] rempli de morceaux écrits aussi purement que s'ils étaient du temps de l'Arioste et du Tasse ${ }^{12}$. Se, come nel caso di Shakesperare, la sua posizione nei riguardi di Dante si fece con il passare degli anni più dura, al punto di applaudire al Bettinelli che in Dante non aveva visto altro che un «fou» e nel suo poema per l'appunto «un monstre» ${ }^{13}$, questo non gli impedì tuttavia di lasciarsi sfuggire, in quella stessa circostanza, questa significativa ammissione: «J'aime encore mieux pourtant dans ce monstre une cinquantaine de vers supérieurs à son siècle, que tant de vermisseaux appelés sonetti, qui naissent et meurent à milliers aujourd'hui en Italie» ${ }^{14}$.

Qualcosa stava quindi cambiando in Francia nei riguardi di Dante. L'uomo era certamente più ammirato di quanto il suo capolavoro non fosse letto. Della Commedia i Francesi avevano una conoscenza ancora molto parziale, quando non, come nel caso di Voltaire, frammentaria; raramente s'andava al di là della prima cantica, o di alcuni episodi (quelli di Paolo e Francesca o del Conte Ugolino), che erano ormai entrati a far parte del patrimonio culturale di molti; tuttavia negli interstizi di un secolo che ufficialmente continuò a ripudiarlo se non ad ignorarlo, l'interesse per Dante e la sua opera si creò, specie nella seconda parte del secolo, varchi sempre più ampi. Ne sono indizi, tutto sommato significativi, gli articoli relativi a Dante che il Chevalier de Jaucourt inserì nell'Encyclopédie o le allusioni alla sua opera che si incontrano in quelle di scrittori come Diderot ${ }^{15}$ e Beau-

(10) «ll me paroît plein de gravité, d'énergie et d'images fortes, mais profondément tristes. Ainsi je n'en lis guères - aveva confessato - car il me rend l'âme toute sombre» (op. cit., p. 890).

(11) [Fr.-TH. BACULARD D'ARNAUD], Les Amants malheureux ou le Comte de Comminge, drame en trois actes et en vers, précédé d'un Discours préliminaire et suivi des Mémoires du Comte de Comminge. A La Haye, et se trouve à Paris, chez L'Esclapart, M. DCC. LXIV, p. VIII. Per Baculard d'Arnaud, il «sombre» prima di rappresentare un colore o un'atmosfera, esprime uno stato d'animo, una tensione spirituale, un modo di rapportarsi alle cose che sono in noi e fuori di noi, un «costume», per usare le sue parole (p. VII), che egli non trova solo nell'arte, semplice e sublime ad un tempo, degli antichi tragediografi greci, ma anche in quella di alcuni grandi artisti moderni, tra i quali, per l'appunto, il Dante dell'Inferno.

(12) VOLTAIRE, Essai sur les mours in ID. Euvres complètes, ed. Beuchot, Paris, Garnier, 1877-1885, t. XII (secondo dell'Essai sur les moeurs), pp. 5859.

(13) Cfr. Lettre sur Dante a Saverio Bettinelli del 1759. Occorre però ricordare che si era nel pieno della polemica scatenata dalle Lettere Virgiliane dell'abate mantovano, nel quale Voltaire aveva trovato un inatteso alleato, di cui prese volentieri, ed in modo senza dubbio eccessivo, le difese contro coloro che in Italia lo avevano pesantemente attaccato.

(14) Ibidem.

(15) D. Diderot, Jacques le Fataliste et son maître, édition critique par Simone LECOINTRE et Jean Le Galdiot, Genève, Droz, 1976, pp. 257258: «LE MAITRE: "Pour moi, je me regarde comme en chrysalide, et j'aime à me persuader que le papillon, ou son âme, venant un jour à percer sa coque, s'envolera à la Justice divine". JACQUES: "Votre image est charmante". LE MAITRE: "Elle n'est pas de moi. Je l'ai lue, je crois, dans un poëte italien appellé Dante, qui a fait un ouvrage intitulé La Comédie de l'Enfer, du Purgatoire et du Paradis". JACQUES: "Voilà un singulier sujet de comédie". LE MAITRE: «"Il y a, pardieu, de belles choses, surtout dans son Enfer». L'allusione è naturalmente ai vv. 124-126 del canto X del Purgatorio ( $\ll$ Non v'accorgete voi che noi siam vermi / nati a formar l'angelica farfalla, I che vola a la giustizia sanza schermi?» ). Dopo essersi chiesti da dove Diderot avesse potuto trarre la citazione, i curatori dell'edizione critica hanno concluso con questa osservazione: «Diderot a très probablement lu la Divine Comédie. Cette lecture correspondrait d'ailleurs à l'intérêt nouveau que suscitait l'œuvre de Dante dans les milieux littéraires français depuis quelques 
marchais ${ }^{16}$, per non parlare di Ducis, che nel suo rifacimento del Romeo and Juliet shakespeariano inserì addirittura l'episodio del conte Ugolino ${ }^{17}$. Un'altra sicura spia del crescente interesse per Dante è rappresentata dalle tre traduzioni che della Commedia, o di una parte di essa, furono intraprese nella seconda metà del Settecento: quella di Colbert d'Estouteville, destinata a rimanere manoscritta fino alla fine del secolo $^{18}$, quella, limitata all'Inferno, di Moutonnet de Clairfons, che vide la luce nel $1776^{19}$, e, soprattutto, quella, sempre dell'Inferno, portata a termine da Antoine Rivarol e apparsa per la prima volta in forma completa nel $1783^{20}$, destinata a segnare una vera e propria svolta nell'atteggiamento dei Francesi nei riguardi di Dante, e a dare avvio a quell'interesse per il capolavoro dantesco che sarebbe sfociato in quell'engouement degli anni 1830-1840, di cui hanno parlato i critici francesi che abbiamo citato più sopra; engouement che, quindi, fu assai meno improvviso, ed inatteso, di quanto, a volte, si tenda o si voglia far credere, proprio perché non è stato dato il giusto peso a questo avvenimento come ad alcuni altri degli anni successivi, sui quali noi insisteremo invece nelle pagine seguenti.

La traduzione, in prosa, di Rivarol all'epoca fu molto criticata; si rimproverò in particolare ad essa l'eccessiva libertà rispetto al testo originale ${ }^{21}$, al punto che più che una traduzione poteva essere definita un adattamento del poema dantesco, esattamente come l'Hamlet di Ducis era un adattamento, assai più che una traduzione dell'omonima tragedia shakespeariana. Rivarol era del resto, come Ducis, troppo figlio del suo tempo, e troppo legato al gusto del suo paese per operare diversamente. Una traduzione letterale sarebbe stata impensabile: la lingua di Dante era troppo grossolana, troppo primitiva per poter entrare nell'elegante sintassi forgiata dal francese; pretendere di tradurla letteralmente avrebbe significato renderla ridicola, e travisare completamente non solo il senso, ma anche lo stile del poema dantesco. Per questo «toutes les fois que le mot à mot n'offrait qu'une sottise ou qu'une image dégoûtante» Rivarol prese «le parti de dissimuler». Se scelse di «s'écarter de son texte» lo fece però «pour [se] coller plus étroitement au Dante même », come lui stesso precisò, convinto che «à cause de ses défauts, Dante exigeait plus de goût que d'exactitude» e che «il fallait avec lui s'élever jusqu'à une sorte de création; ce qui forçait le traducteur à un peu de rivalité» ${ }^{22}$. Più che una traduzione dell'Inferno dantesco, il suo Enfer si pre-

années» (p. 453). Pubblicato solo nel 1796, Jacques le fataliste era stato iniziato nel 1765 e composto per la maggior parte negli anni successivi.

(16) Beaumarchais aveva quasi certamente in mente Dante quando, nel corso del celebre monologo del V atto del Mariage, fece dire a Figaro: «Sitôt je vois, du fond d'un fiacre, baisser pour moi le pont d'un château fort, à l'entrée duquel je laissais l'espérance et la liberté».

(17) Cfr. Roméo et Juliette, tragédie par M. Ducis, représentée pour la première fois par les Comédiens François Ordinaires du Roi, le 17 Juillet 1772. A Paris, chez P. F. Gueffier, M. DCC. LXXII, pp. 54-55 (atto IV, scena V).

(18) Cfr. La Divine Comédie de Dante Alighieri, contenant la description de l'Enfer, du Purgatoire et $d u$ Paradis, traduite de l'Italien par le comte P.-E. Colbert, Duc d'Estouteville, revue par M.-F. Sallior, éditeur, précédée de la Vie de Dante de Bullart. Paris, chez Sallior, successeur de Didot le Jeune, l'an IV de la République [1796]. Marc Scialom (op. cit., p. 127) fa osservare che «M.-F. Sallior a profondément modifié cette traduction, dont il existe par ailleurs trois mss».

(19) La Divine Comédie, L'Enfer, traduction françoise accompagnée du texte, de notes historiques, critiques \& de la Vie du poëte, par M. MouTONNET DE Clairfons, Florence et Paris, Le Clerc, Le Boucher, 1776.

(20) Cfr. L'Enfer, poëme du Dante, traduction nouvelle, par le comte A. DE RIVAROL, avec un mémoire sur le Dante par De Mérian. Londres, Paris, Mérigot Le Jeune et Barrois, 1783.

(21) Cfr. in particolare l'articolo che NicolasEtienne Framery pubblicò sul «Mercure de France» del 25 giugno 1785, pp. 153-173, al quale Rivarol rispose sulle colonne del «Journal de Paris» del 29 luglio 1785 , pp.867-868.

(22) A. Rivarol, L'Enfer, poème du Dante, cit., in Euvres complètes de Rivarol. A Paris, 1806, t. III, pp. 164-165. L'osservazione è già stata fatta da Marisa FERRARINI nel suo contributo Dante, Rivarol o Moutonnet? in La nascita del concetto moderno di traduzione. Le nazioni europee tra enciclopedismo e epoca romantica, a cura di Gabriella CATALANO e Fabio Scotto, Roma, Armando editore, 2001, p. 217. 
sentò quindi come una sorta di «recréation», fatta in quello «style soutenu» richiesto da Voltaire, e più in generale, dal gusto classico, il quale se apparentemente poteva non avere nulla a che fare con il ruvido dettato dantesco, in realtà era, a parere del Rivarol, quello che meglio poteva permettere ai Francesi del suo tempo, di gustare le bellezze e la forza dello stile messo a punto da Dante, in un'epoca in cui la lingua italiana era ai suoi primordi. D'altra parte, fece ancora notare Rivarol, «il fallait que le Dante pour produire tout son effet, se présentât dans notre langue tel qu'il s'offrit autrefois dans la sienne» ${ }^{23}$.

Si trattò, ad ogni modo, di uno sforzo notevole, degno della massima attenzione che alcuni dei migliori critici dell'epoca riconobbero volentieri a Rivarol. Melchior Grimm fece, per esempio, osservare sulla sua Correpondance littéraire:

Quoique le ton de cette nouvelle traduction ne soit pas également soutenu, quoiqu'elle nous ait paru manquer souvent tout à la fois d'élégance et de fidélité, nous y avons trouvé de grandes difficultés heureusement vaincues, et n'en déplaise à l'ineptie ou à la sévérité de ceux qui l'ont critiquée avec tant d'acharnement, nous osons penser qu'elle est bien supérieure à toutes celles que nous connaissions. La physionomie du Dante, l'odeur de son siècle y transpirent du moins à chaque page $e^{24}$.

Ancora più significativo è il giudizio che al riguardo espresse oltre mezzo secolo più tardi, quindi in piena epoca romantica, Augustin de Sainte-Beuve: «Honneur à Rivarol! On dira de sa traduction tout le mal qu'on voudra, on ne lui enlevera pas le mérite d'avoir le premier chez nous apprécié avec élévation la nature et la qualité du génie de Dante» ${ }^{25}$.

Pubblicata per la prima volta nel 1783, ristampata due anni più tardi da Didot le Jeune e ripresa nel 1788 dalla Bibliothèque Universelle des Romans, che ne aveva anticipato i brani più significativi nel $1780^{26}$, la traduzione di Rivarol ebbe, di fatto e fin dall'inizio, e nonostante gli innegabili difetti, un notevole impatto. Come ebbe giustamente ad osservare Albert Counson, il primo storico della fortuna di Dante al di là delle Alpi, «Rivarol fit faire un grand pas à la connaissance de Dante» al di là delle Alpi, precisando che con Rivarol cominciò «bien quelque chose de neuf, puisque nous retrouverons des traces de son ouvrage par la suite» ${ }^{27}$. Occorre anche dire che nell'«Avis de l'Editeur», che precede la traduzione, Rivarol aveva saputo richiamare l'attenzione dei suoi concittadini sulla straordinaria importanza che l'opera di Dante aveva avuto ed ancora aveva sull'arte europea. «Il n'est guère dans la littérature de nom plus imposant que celui du Dante - aveva infatti scritto Rivarol, che più oltre aveva fatto notare come «le génie d'invention, la beauté des détails, la grandeur et la bizarrerie des conceptions» avessero meritato a Dante «je ne dis pas la première ou la seconde place entre Homère et Milton, le Tasse et Virgile, mais une place à part» ${ }^{28}$. Rivarol, per altro verso, aveva inserito molto bene, per l'epoca, l'opera di Dante nel tempo e nel contesto in cui il poeta era vissuto ed aveva operato:

Quoique le génie n'attende pas des époques pour éclore, supposons cependant - faceva infatti notare - que dans un siècle effrayé par tant de catastrophes, et dans le pays même théâtre de tant de discordes, il se rencontre un homme de génie qui, s'élevant au milieu des orages, parvienne au gouvernement de sa patrie; qui ensuite exilé par des citoyens ingrats, il soit réduit à

(23) A. RivaroL, L’Enfer cit., p. 227. Nota al canto XX.

(24) Correspondance littéraire, août 1785.

(25) Causeries du lundi, Paris, Garnier, 1943, t. XI, p. 169. Feuilleton dell'11 dicembre 1854.

(26) L'Enfer, poème du Dante, in Bibliothèque
Universelle des Romans, Paris, Janvier 1780. Vol. II, 1ère classe: «Romans étrangers», pp. 1-64 (Inferno, I-VII).

(27) A. Counson, Dante en France cit., p. 95. p. XLIV. 
traîner une vie errante, et à mendier le secours de quelques petits souverains; il est évident que les malheurs de son siècle et ses propres infortunes feront sur lui des impressions profondes, et le disposeront à des conceptions mélancoliques et terribles. Tel fut le Dante, qui conçut dans l'exil son poème de l'Enfer, du Purgatoire et du Paradis embrassant dans son plan les trois règnes de la vie future, et s'attirant toute l'attention d'un siècle où on ne parlait que du jugement dernier, de la fin de ce monde, et de l'avenement d'un autre ${ }^{29}$.

Sebbene egli avesse limitato la sua fatica di traduttore all'Inferno, era dunque, a differenza di quanto era successo fino ad allora, su tutto il grande poema dantesco che Rivarol attirò l'attenzione dei suoi contemporanei.

Un altro passo importante, se non decisivo, sulla strada della riscoperta di Dante da parte dei Francesi, fu compiuto, poco oltre quella Rivoluzione che, se sconvolse l'assetto sociale e politico della Francia, non cambiò nulla, o quasi, dal punto di vista letterario, ad opera di Pierre-Louis Ginguené, un uomo sul quale la critica aveva fino a qualche tempo fa sorvolato un po' troppo, e del quale gli studi condotti in questi ultimi anni hanno invece posto in evidenza la straordinaria importanza nel campo della storia letteraria in generale e del ruolo da lui svolto per una più approfondita conoscenza di Dante in particolare. Nato a Rennes nel 1748, Ginguené fu, come Rivarol, uno dei tanti giovani provinciali che venne a Parigi in cerca di fortuna e di onori, e che nella capitale si arruolò presto tra le file di quegli Illuministi che più tardi si chiameranno Idéologues. Egli fu però soprattutto un profondo conoscitore dell'Italia, di cui apprese ben presto la lingua e di cui studiò con passione e competenza crescenti la storia, la musica e sopratutto la letteratura; alla quale, pur essendo figlio del suo tempo e quindi del gusto francese, si accostò con un atteggiamento molto diverso da quello, poniamo, di un Laharpe abituato a trarre le sue analisi da principi assoluti, nella consapevolezza che ogni opera è strettamente legata al momento che l'ha vista nascere, per cui autori ed opere devono, per poter essere colti nella loro importanza e nel loro giusto significato, essere situati «dans le cadre qui leur est propre» ${ }^{30}$.

È quanto egli tentò di fare, a partire dall'anno accademico 1802-1803, nell'ambito della cattedra di Histoire littéraire moderne creata per lui all'Athénée di Parigi e che egli occupò, con una breve interruzione, fino al 1807. Dopo un ciclo di conferenze volte ad illustrare lo sviluppo della civiltà occidentale dall'estinzione del mondo classico fino al sorgere delle letterature nazionali, Ginguené procedette allo studio della letteratura italiana, che seguì dai primi componimenti in volgare del Duecento fino alla lirica del Cinquecento. Qualche anno più tardi Ginguené raccolse e sistemò quelle lezioni in un'opera, l'Histoire littéraire d'Italie, che nella mente del suo autore doveva essere la prima parte di un lavoro assai più ampio, ma del quale egli non riuscì a pubblicare in realtà che i primi sei volumi, usciti dal 1811 al 1813: abbastanza tuttavia per fare dello Ginguené non solo l'Italianisant più competente del suo tempo ma anche, come si diceva, il più importante ed efficace presentatore di Dante in terra francese; con un'opera che non si rivolgeva più solo agli eruditi, che si apriva invece ad un pubblico volutamente più vasto ed eterogeneo, che andava dagli amateurs e dalle gens du monde fino al nuovo pubblico che il secolo dei lumi e gli ultimi rivolgimenti politico-sociali avevano portato alla ribalta. La forma, volutamente snella e moderna, poggiava non solo su una profonda conoscenza della migliore letteratura

(29) Ibid., p. XVIII.

(30) P.-L. GingueNÉ, Recensione a Le Lycée ou Cours de Littérature ancienne et moderne, par J.F. LAHARPE. A Paris, Chez Agasse, in «La Décade philosophique, littéraire et politique» t. XXII, 10 fructidor an VIII (27/8/1799), p. 424. Cit. tratta da C. Trinchero, Pierre-Louis Ginguené (1748-1816) e l'identità nazionale italiana nel contesto culturale europeo, Roma, Bulzoni, 2004, p. 45. 
critica, sopratutto italiana, di cui lo studioso poteva allora disporre ma anche su una conoscenza altrettanto profonda e seria dei diversi autori e delle diverse opere. Questo vale soprattutto per Dante al quale Ginguené dedicò oltre trecento pagine della sua Histoire, vale a dire quasi un intero volume, a segno non solo della cura con la quale egli volle presentare ai suoi concittadini il grande poeta italiano, ma anche dell'importanza che a Dante egli conferiva nel contesto della letteratura italiana.

Ginguené parla di Dante dopo aver ricordato l'«Etat des Lettres en Italie au treizième siècle». Inizia con una «notice abrégée de sa vie, dont les vicissitudes sont liées aux événements politiques de son temps $\gg^{31}$, e prosegue con una rapida ma puntuale analisi delle opere minori, «bien inférieures sans doute» alla Commedia ma che è bene aver presenti perché «rien de ce qui est sorti d'un génie de cet ordre n'est indifférent pour l'histoire des lettres ${ }^{32}$. È solo dopo aver inserito il poeta nel contesto storico e sociale del suo tempo ed aver presentato le sue opere minori che Ginguené analizza il suo capolavoro: lo fa in tre lunghi capitoli consacrati ognuno ad una delle tre cantiche in cui il poema è suddiviso. Certo, il capitolo dedicato all'Inferno è sensibilmente più lungo di quello dedicato al Purgatorio e, soprattutto, di quello dedicato al Paradiso. Le preferenze di Ginguené, come quelle dei suoi contemporanei, vanno ancora chiaramente alla prima cantica e agli episodi che la tradizione aveva trascelto, anche se per motivi diversi: quelli di Paolo e Francesca e del conte Ugolino in primis. Tuttavia il tentativo di Ginguené di presentare ai lettori francesi l'opera di Dante nella sua interezza, tramite un'analisi che si sforza di essere oggettiva, è lodevole, e risponde al desiderio di far conoscere l'opera del grande poeta nella sua totalità e nella sua complessità; anche nella sua indubbia difficoltà. Per apprezzare la poesia di Dante, avverte Ginguené, occorre che il lettore francese esca dai suoi schemi mentali, dalle sue abitudini, che faccia uno sforzo. Questo vale per l'episodio del conte Ugolino («Une fausse délicatesse peut trouver dans ces vers et dans leur traduction une espèce de crudité de style, mais ce n'est ni au Dante ni à sa langue qu'il faut la reprocher, c'est à nous et à la nôtre», si legge in una nota di commento ai versi più crudi dell'episodio ${ }^{33}$ ), come per i versi apparentemente più aridi del Paradiso, che il lettore deve sforzarsi di leggere come un'opera di poesia, consiglia Ginguené ${ }^{34}$, piuttosto che seguire i commentatori nei loro tentativi, più o meno convincenti, di spiegare il significato delle varie allegorie alle quali Dante è ricorso, qui ancor più spesso che altrove. Per illustrare meglio la Commedia Ginguené ricorre spesso, soprattutto per la prima cantica, ad ampie citazioni che traduce quasi sempre egli stesso cercando la maggiore aderenza al testo originale, ed anche questa è cosa che merita di essere sottolineata: alle «belles infidèles» di Rivarol succedono traduzioni progressivamente sempre più attente a rendere non solo il senso ed il tono, ma anche la forma, pur nella consapevolezza che, con Dante, la cosa non è sempre facile. Ne risulta una presentazione che mette bene in evidenza non solo la grandiosità della «machine poétique» inventata da Dante $^{35}$, ma anche la straordinaria potenza della sua esecuzione:

Doué d'un génie vaste, d'un esprit pénétrant et d'une imagination ardente, il joignit à des connaissances étendues une vivacité de pensées, une profondeur de sentiment, un art d'employer d'une manière neuve des expressions communes, et d'en inventer des nouvelles, un

(31) Cfr. Histoire littéraire d'Italie, par P.-L. GINGUENÉ, membre de l'Institut de France. A Paris, chez Michaud frères, M. DCCC. XI, t. I, p. 437.

(32) Ibid., t. I, p. 458.

(33) Ibid., t. II, p. 119.

(34) «Suivons donc le poète et sa conductrice, et ne choisissons d'autres détails dans leur dernier voyage, que ceux qui s'accordent avec l'objet pure- ment littéraire qui nous l'a fait entreprendre avec eux». Ibid., t. II, p. 198.

(35) Ibid. t. II, p. 2. Alla fine dell'analisi-presentazione del poema, Ginguené ribadisce il concetto: «Après l'avoir suivi dans ce voyage, d'aussi près que nous l'avons fait, nous sommes plus en état qu'on ne l'est d'ordinaire, d'en apprécier la marche hardie et l'étonnante exécution» (ibid., t. II, p. 252). 
talent de peindre et d'imiter, un style serré, vigoureux, sublime, qui, malgré les défauts, qu'on ne doit imputer qu'au temps où il vécut, lui ont toujours conservé la place que lui décerna l'admiration de son siècle ${ }^{36}$.

I difetti certo non mancano nell'opera di Dante e Ginguené, sulla scorta di alcuni degli storici italiani più accreditati e sulla scia delle sue mai rinnegate radici classiche, non ha difficoltà ad ammetterlo:

Le plus grand défaut, dans l'ensemble, est de manquer d'action [...[ Où manque une action principale, il n'y a de point d'appui que les épisodes, et un poème tout en épisodes ne peut ni soutenir toujours l'attention, ni ne la pas fatiguer quelquefois. Le défaut le plus choquant dans les détails est peut-être ce mélange, cet accozzamento, comme disent les Italiens, de l'antique et du moderne, et de l'Histoire sainte avec la Fable. L'obscurité en est un autre qui n'est pas moins importun ${ }^{37}$.

Tuttavia, se si tiene conto del tempo nel quale il grande poema è stato ideato e delle precarie condizioni in cui è stato composto, non si può non restare ammirati degli straordinari risultati ai quali le grandissime doti poetiche hanno consentito a Dante di arrivare:

Dans un siècle si reculé, après une si longue barbarie et de si faibles commencements, on est surpris de voir la poésie et la langue prendre une démarche si ferme et un vol si élevé - fa osservare Ginguené -. Dans ses vers on voit agir et se mouvoir chaque personne, chaque objet qu'il a voulu peindre. L'énergie de ses expressions frappe et ravit; leur pathétique touche; quelquefois leur fraîcheur enchante; leur originalité donne à chaque instant le plaisir de la surprise. Ses comparaisons fréquentes et ordinairement très courtes et arrondies, comme celle d'Homère, tantôt nobles et élevées, tantôt communes et prises même des objets les plus bas, toujours pittoresques et poétiquement exprimées, présentent un nombre infini d'images vives et naturelles, et les peignent avec tant de vérité qu'on croit les avoir sous les yeux ${ }^{38}$.

È difficile valutare l'azione svolta dallo Ginguené a favore di una migliore conoscenza della letteratura italiana, e di Dante in particolare, in Francia. Assai più importante di quanto fino a poco tempo fa si fosse creduto, essa fu certamente più significativa ed efficace di quella esercitata, allo stesso fine, dal fin troppo conclamato Chateaubriand che, all'epoca in cui compose il Génie du Christianisme di Dante aveva, in realtà, una conoscenza piuttosto limitata e, cosa più grave, distorta da evidenti pregiudizi di ascendenza classico-settecentesca, per cui la Divina Commedia era ancora, ai suoi occhi, «cette production bizarre» le cui «beautés découlent presque entièrement du christianisme», mentre «ses défauts tiennent au siècle et au mauvais goût de l'auteur» ${ }^{39}$; essa fu, probabilmente, superiore anche a quella esercitata da

(36) Ibid.t. I, p. 457.

(37) Ibid., t. II, pp. 258-259.

(38) Ibid., t. II, p. 260.

(39) F.-R. de Chateaubriand, Génie du Christianisme, éd. Louis Lover, Paris, Garnier, 1939, p. 146. Della scarsa considerazione, frutto di un'insufficiente conoscenza, che lo Chateaubriand aveva del poema dantesco si rese lucidamente conto lo Ginguené il quale, nella recensione del Génie du christianisme pubblicata sulla «Décade philosophique» del 10 messidor an X (29 giugno 1792) scrisse: «Il [Chateaubriand] fait d'abord une revue des principaux poëmes où le merveilleux du christianisme remplace la mythologie; et le premier qui s'offre à lui est celui de Dante. On est surpris qu'il n'en parle qu'en douze lignes, et seulement pour dire qu'il n'en dira rien. Son prétexte est que ce poëme, étant d'une nature toute épisodique, soutiendrait mal une analyse régulière. Il promet de revenir sur les détails, et il n'y revient dans la suite que pour rappeler deux ou trois principaux traits connus de tout le monde. Tout prouve que cette production originale, qui est à la vérité très peu connue et très mal appréciée en France, lui est entièrement étrangère». Cfr. S. ZoPPI, P.L. Ginguené journaliste et critique littéraire. Textes choisis avec une introduction et des notes, Torino, Giappichelli, 1968, p.149. 
Madame de Staël, che scoprì la Commedia dantesca sotto la guida del Parini al tempo del suo viaggio in Italia e che in Corinne ou de l'Italie, apparsa nel 1807, mise in bocca alla protagonista un'entusiastica presentazione di Dante:

Le Dante, l'Homère des temps modernes, poète sacré de nos mystères religieux, héros de la pensée, plongea son génie dans le Styx, pour aborder à l'enfer, et son âme fut profonde comme les abîmes qu'il a décrits. L'Italie, au temps de sa puissance, revit tout entière dans le Dante. Animé par l'esprit des républiques, guerrier aussi bien que poète, il soumit la flamme des actions parmi les morts, et ses ombres ont une vie plus forte que les vivants aujourd'hui. Les souvenirs de la terre les poursuivent encore; leurs passions sans but s'acharnent à leur cœur; elles s'agitent sur le passé, qui leur semble encore moins irrévocable que leur éternel avenir. On diroit que le Dante, banni de son pays, a transporté dans les régions imaginaires les peines qui le dévoroient. Ses ombres demandent sans cesse des nouvelles de l'existence, comme le poète lui-même s'informe de sa patrie, et l'enfer s'offre à lui sous les couleurs de l'exil ${ }^{40}$.

Certamente più celebre e nota, non fosse che per il nome dell'autrice e per il grande successo che il libro che la conteneva riscosse in tutta Europa, l'evocazione di Madame de Stael è anche, occorre dirlo, più limitata e soggettiva. Se nel seguito, la protagonista fa riferimento anche al Purgatorio ed al Paradiso, il Dante di Mme de Staël è prima di tutto il poeta dell'Inferno e dell'esilio. Nel grande Toscano la castellana di Coppet vede essenzialmente un fratello maggiore che prima di lei ha subito il bando dalla propria città e che, come lei, spera che la fama letteraria gli possa valere il ritorno ${ }^{41}$. Se questo non impedisce certo a Madame de Staël di ammirare ed apprezzare al suo giusto valore il poeta:

A sa voix tout sur la terre se change en poésie; les objets, les idées, les lois, les phénomènes semblent un nouvel Olympe de nouvelles divinités [...] Les magiques paroles de notre plus grand poète sont le prisme de l'univers; toutes ses merveilles s'y réfléchissent, s'y divisent, s'y recomposent; les sons imitent les couleurs, les couleurs se fondent en harmonie; la rime, sonore ou bizarre, rapide ou prolongée, est inspirée par cette divination poétique, beauté suprême de l'art, triomphe du génie, qui découvre dans la nature tous les secrets en relation avec le cœur de l'homme» fa osservare Corinne per conto di Madame de Stä̈l, ${ }^{42}$

per l'autrice di Corinne Dante è prima di tutto il poeta dell'esilio e dell'impegno politico. Come ha fatto giustamente osservare A. Counson, Madame de Staël «cherche à se retrouver et à se mirer dans le portrait de Dante; elle aussi est exilée, elle aussi espère en sa gloire littéraire» ${ }^{43}$. Quella che Madame de Staël ci offre di Dante è una lettura per certi versi più «romantica» che critica; per questo non ci pare di poter condividere la conclusione di Michael Pitwood secondo cui «the view of Dante which emerges [da Corinne] is both more positive and more balanced than any which preceded it in France», mentre ci sembra più rispondente al vero la riflessione che lo stesso critico aveva fatto poco prima, secondo cui «Corinne's treatement of Dante is the product of subjective enthusiasm rather than empirical analysis» e che «her comments are mostly couched in general terms $\gg^{44}$; con un atteggiamento, quindi, molto diverso da quello dello Ginguené, che non rimase isolato.

(40) Madame de StaëL, Corinne ou l'Italie, éd. Claudine Hermann, Paris, Editions des Femmes, 1979, t. I, p. 53 (Livre II: «Corinne au Capitole», chapitre 3 )

(41) «Le Dante espéroit de son poëme la fin de son exil, il comptoit sur la renommée pour média- teur, mais il mourut trop tôt pour recueillir les palmes de la patrie» (Ibid., p. 54).

(42) Ibid., p. 54.

(43) A. Counson, Dante en France, cit., p. 112.

(44) M. Pitwood, Dante and the French Romantics, cit., pp. 73-74. 
Ginguené trovò infatti un continuatore, ed un imitatore, in un personaggio che faceva parte del gruppo di intellettuali e di letterati che si riunivano a Coppet attorno a Madame de Staël: si tratta del ginevrino Simonde de Sismondi che a Dante consacrò una quarantina di pagine del suo libro De la littérature du midi de l'Europe. Pur essendo partito da presupposti molto vicini a quelli che erano stati elaborati dal gruppo di Coppet («J'ai surtout voulu montrer partout l'influence de l'histoire politique et religieuse des peuples sur leur littérature, et de leur littérature sur leur caractère» ${ }^{45}$ ), nella presentazione di Dante il Ginevrino seguì abbastanza da vicino lo Ginguené, del quale citò peraltro a più riprese e con elogio l'Histoire littéraire de l'Italie. Iniziò anche lui inserendo Dante nel contesto storico e letterario del suo tempo, prima di sottolineare la sua straordinaria grandezza e la sua unicità, in particolare per la sua capacità di trasformare una lingua ancora rozza e primitiva in uno strumento di grande duttilità e di straordinaria forza poetica:

Aucun poète n'avait encore remué fortement les âmes, aucun philosophe n'avait pénétré dans les profondeurs de la pensée et du sentiment, lorsque le plus grand des Italiens, le père de leur poésie, lorsque le Dante parut, et qu'il montra comment un puissant génie pouvait disposer ces matériaux grossiers encore, de manière à en construire un édifice imposant comme l'univers dont il était l'image. Au lieu de chants d'amour, adressés à une maîtresse imaginaire, au lieu de madrigaux froidement spirituels, de sonnets péniblement harmonieux, d'allégories fausses ou forcées, seuls modèles que le Dante eut sous les yeux dans aucune langue moderne, il conçut dans son cerveau tout le monde invisibile, et il le dévoila aux yeux de ses lecteurs étonnés ${ }^{46}$.

Il capolavoro dantesco è presentato attraverso un'analisi dei momenti più importanti, illustrati, come nell'Histoire dello Ginguené, da citazioni tradotte nello stesso senso del suo predecessore, con una netta preferenza per l'Inferno, rispetto al Purgatorio, di cui Sismondi fatica a cogliere la delicata poesia, e soprattutto del Paradiso, il più lontano dai suoi gusti in quanto troppo infarcito di discussioni teologiche $\mathrm{e}$ filosofiche, e troppo povero invece di quelle descrizioni che tanto aveva apprezzato nell'Inferno. Questo non impedisce però al Sismondi di concludere la sua presentazione del capolavoro dantesco con un apprezzamento altrettanto entusiasta di quello con il quale lo Ginguené aveva concluso la sua:

Peu de chefs-d'œuvre ont mieux manifesté la force de l'esprit humain que le poëme du Dante: complètement nouveau dans sa composition comme dans ses parties, sans modèle dans aucune langue, il était le premier monument des temps modernes, le premier grand ouvrage qu'on eût osé composer dans aucune des littératures nouvellement nées. Il était conforme aux règles essentielles de l'art, à celles qui sont invariables: l'unité de dessein, l'unité de marche, l'empreinte d'un génie puissant qui voit en même temps le tout et ses parties, qui dispose avec facilité des plus grandes masses, et qui est assez fort pour observer la symétrie sans en ressentir jamais de gêne. A tout autre égard le poëme du Dante était en dehors des anciennes règles de l'art poétique, il n'appartenait proprement à aucun genre, et le Dante ne pouvait être jugé que par les lois qu'il s'était données ${ }^{47}$.

Se la posizione critica assunta dal Sismondi non era molto originale, la sua opera, che ebbe un certo successo in Francia negli anni seguenti, contribuì certamente, al pari di quella dello Ginguené, alla quale, per quanto riguarda Dante almeno, essa si

(45) J.C.L. Sismonde De Sismondi, De la Littérature du Midi de l'Europe. A Paris, chez Treuttel et Wurtz, 1813, t. I, p. II.
(46) Ibid. p. 350.

(47) Ibid., p. 386. 
era sicuramente ispirata, a far fare all'interesse per il poeta italiano un altro significativo passo avanti.

Certamente più efficace, in questo senso, fu però la traduzione della Commedia pubblicata in quegli stessi anni da Alexis-François Artaud de Montor, un altro di quei preziosi, anche se un po' oscuri operai sui quali la critica ha spesso sorvolato e che rappresenta invece un'altra delle tappe fondamentali sulla strada della (ri)scoperta di Dante da parte dei Francesi. Alexis-François Artaud de Montor era un diplomatico di carriera che le vicende della vita condussero dapprima a Roma poi a Firenze. A Dante si accostò, come egli stesso ebbe a confessare, verso il 1805 «avec le secours d'habiles Florentins», tra i quali un ruolo fondamentale ebbe l'abate Fontani, che non solo gli fecero conoscere la Commedia ma che lo introdussero anche, progressivamente, nei più reconditi segreti del poema che a poco a poco divenne per Artaud de Montor la ragione stessa della sua esistenza. Di qui il suo desiderio di farlo meglio conoscere ai suoi concittadini grazie ad una traduzione che fosse il più possibile fedele al testo dantesco e tenesse conto dei risultati della più recente ed accreditata ricerca filologica. Per la sua traduzione Artaud de Montor scelse, come già aveva fatto Rivarol, per il quale non nasconde la sua ammirazione, la prosa, ma a differenza del suo predecessore, egli cercò, come dicevamo, di rendere quanto più fedelmente fosse possibile il testo dantesco, che corredò peraltro di tutta una serie di note e di commenti tratti dagli studi più recenti, al fine di rendere la comprensione ancora più precisa. Ne venne fuori una traduzione per il vero un po' laboriosa, per certi aspetti anche savante, fedele tuttavia, per quanto era allora possibile, al testo dantesco, che incontrò subito un grande successo presso il pubblico; non tanto, forse, per le sue qualità quanto per il crescente bisogno che i Francesi avvertivano di accostarsi alla Commedia tramite una traduzione che non solo rendesse il tono o la sostanza del capolavoro dantesco, ma tentasse di renderne anche il dettato. Artaud de Montor iniziò significativamente dal Paradiso, vale a dire dalla Cantica meno conosciuta e che lui aveva invece imparato ad apprezzare più delle altre due, la cui traduzione egli pubblicò negli anni successivi ${ }^{48}$.

La traduzione dell'Artaud segnò una tappa molto importante nella storia della scoperta di Dante da parte dei Francesi ed essa aprì, assai più di quanto all'inizio del secolo scorso intendesse dire A. Counson, «une ère [davvero] nouvelle dans la dantographie française $»^{4}$. Occorre intanto far osservare che lo sforzo intrapreso, alcuni anni prima, dallo Ginguené di presentare ai Francesi la Commedia nella sua interezza trovò proprio nella traduzione dell'Artaud la sua degna conclusione. E non a caso, proprio dallo Ginguené venne il primo importante riconoscimento della qualità e dell'importanza della traduzione curata e messa a punto dal diplomatico francese:

Cette traduction, exacte, et qui rend, autant peut-être qu'il est possibile, d'après la différence des deux langues, les beautés de l'original, est accompagnée de notes très utiles pour l'intelligence du texte, pour l'explication des allégories, des faits historiques et des difficultés de langue. Il ne paraît pas que le plus grand poète de l'Italie puisse espérer en France un meilleur traducteur

(48) Le Paradis poëme de Dante, traduit de l'italien, précédé d'une Introduction, de la Vie du poëte, et d'un Catalogue de 80 éditions de la Divine Comédie de cet Auteur; suivi de Notes explicatives pour chaque chant; par un Membre de la Société Colombaire de Florence [...] . A Paris, chez Treuttel \& Würtz, 1811; L'Enfer, poëme de Dante, suivi de Notes explicatives pour chaque chant; par un
Membre de la Société Colombaire de Florence. A Paris, chez J. Smith et F. Schoell, 1812; Le Purgatore, poëme de Dante, traduit de l'Italien, suivi de notes explicatives pour chaque chant, par un Membre de la Société Colombaire de Florence, Paris, J.-J. Blaise, 1813.

(49) A. Counson, Dante en France, cit., p. 118. 
scrisse Ginguené nel 1813 nella voce consacrata a Dante nella nuova Biographie universelle edita dal Michaud ${ }^{50}$. Ginguené non fu comunque l'unico che sottolineò l'importanza dell'operazione intrapresa dall'Artaud. In occasione della riedizione dell'Enfer, nel 1829, la «Quotidienne», un foglio che, pur essendo aperto alla nouvelle école, non stravedeva per Dante, faceva infatti notare come la traduzione dell'Artaud si distinguesse da tutte quelle che l'avevano preceduta «par une plus parfaite intelligence du texte, par un coloris plus vrai, enfin par ces notions toutes locales, que le génie lui-même ne saurait suppléer», e come «quoiqu'habilement adouci, le cynisme grotesque de quelques détails» non fosse «pas même oublié; car suivant sa propre expression, M. Artaud amène le lecteur français jusqu'où l'on peut le conduire sans lui manquer de respect», concludendo che «c'est donc sa traduction que doivent lire les personnes jalouses de connaître le Dante tout entier, et de se former une idée précise de son système de composition $»^{51}$.

Di fatto, sulla traduzione dell'Artaud, uscita per la prima volta in tre grossi volumi con testo originale a fronte dal 1811 al 1813 e riprodotta a varie riprese ed in vari formati negli anni successivi ${ }^{52}$, conobbero Dante non solo Lamartine che, ad anni di distanza, ha tessuto dell'uomo e della sua opera lodi perfino esagerate ${ }^{53}$, ma un'intera generazione di Francesi; quella stessa che a partire dagli anni Trenta diede vita alla breve ma intensa stagione del Romanticismo più vivace e cosciente, e che ebbe in Dante uno dei suoi vessilli più significativi, al punto da farne una sorta di icona e di trasformare presto l'ammirazione in vero e proprio engouement; quello stesso di cui ha parlato J. Risset, e che hanno così ben ricostruito, seppure in forme ed in prospettive diverse, Albert Counson all'inizio del secolo scorso, e Michael Pitwood, in anni a noi più vicini. In realtà, la traduzione dell'Artaud ebbe anche un altro effetto: essa fu infatti all'origine di tutta una serie di traduzioni, più o meno parziali, che videro la luce negli anni successivi. Lo ricordò già nel 1840 Gragnier de Cassagnac sulla «Revue des deux mondes» in un articolo, per la verità non molto benevolo nei riguardi dell'Artaud, del quale riconosceva tuttavia lo scrupolo, oltre che l'importanza per la conoscenza di Dante in Francia. Dopo aver fatto, con un certo disprezzo, i nomi di Gourbillon, di Terrasson, di Chabanon e di Le Dreuil ${ }^{54}$, Gragnier de Cassagnac faceva anche quello di Antoni Deschamps. Ora Antoni Deschamps, poeta oggi quasi del tutto dimenticato, attorno agli anni 28-30 dell'Ottocento, prima che la pazzia lo

(50) [P.-L. GinguenÉ], «Dante Alighieri», in Biographie universelle ancienne et moderne, par une société de gens de lettres, A Paris, chez Michaud Frères, 1813 , t. X, p. 523.

(51) E. GERARD, Recensione a L'Enfer de Dante Alighieri, traduit en français, par M. le chevalier ARTAUD, ancien chargé d'affaires de France à Florence, à Vienne et à Rome. Seconde édition. De l'imprimerie de Firmin Didot, imprimeur du Roi, rue Jacob, n. 24, in «La Quotidienne» del 12 agosto 1829 , p. 4.

(52) Cfr. La Divine Comédie de Dante Alighieri, traduite en français par M. le Chevalier A.-F. ARTAUD, Paris, A. Firmin-Didot, 1812, 9 voll.; L'Enfer de Dante Alighieri, traduit en français par M. le Chevalier A.-F. Artaud, Paris, A. Firmin-Didot, 3 voll.; La Divine Comédie de Dante Aligieri, Enfer, Purgatore et Paradis, traduite de l'Italien par A.-F. ARTAUd De MonTor, avec le texte en regard, et suivi de notes, Paris, A. Firmin-Didot, 1828-1830, 9 voll. Notizie tratte da M. SCIALOM, op. cit., passim.

(53) Dopo aver ricordato le lunghe ed appassio- nate conversazioni avute nella sua giovinezza con Artaud de Montor a proposito di Dante, Lamartine prosegue così nei suoi ricordi: «Ce souvenir m'a peut-être rendu partial pour sa traduction et pour son commentaire; mais j'avoue que jusqu'ici je n'ai pu lire avec une complète sécurité de sens le poème du Dante que dans l'édition en deux langues de M. Artaud, et en contrôlant à chaque instant le texte par le commentaire. M. Artaud n'était pas poète, j'en conviens; mais il était savant. Dante était assez poète pour deux». Cfr. A. DE LAMARTINE, «Traducteurs et commentateurs du Dante», in ID., Souvenirs et portraits, Paris, Hachette, 1872, t. III, pp. 164-165.

(54) Cfr. L'Enfer poème de Dante Alighieri, traduit en vers français, avec des notes. Suivi de traductions, imitations et poésies diverses, par Henri TERRASSON, Paris, Pillet, 1817; Dante, traduit en vers par stances correspondantes aux tercets textuels, sur un texte nouveau quant au choix des variantes et au mode de ponctuation, dédié au Roi, par Joseph Antoine de Tourbillon, L'Enfer, Paris, A. Auffray, 1831. 
costringesse, come più tardi Nerval, a trovare rifugio nella famosa clinica del Docteur Blanche, fu non solo uno degli uomini di punta del Cénacle, e più in generale del movimento romantico francese, ma anche l'autore di una celebratissima, ancorché parziale, traduzione della Commedia dantesca che costituì certamente uno dei motivi più importanti di quell'engouement che il Romanticismo francese conobbe, a partire dal 1830, per Dante. La sua traduzione, apparsa alla fine del 1829, si volle assolutamente diversa da quelle che l'avevano preceduta. Lo scrisse lui stesso nella Préface: «En traduisant la Divine Comédie, nous avons voulu faire une œuvre d'art, non un travail d'érudition, et nous nous sommes proposé avant tout de donner une idée du ton et de la manière de Dante; c'est pourquoi nous publions ces vingt chants, sans notes et commentaires; or pour qui ne cherchera en nous lisant que ce que nous avons cherché en écrivant, c'est-à-dire l'esprit et le style Dantesques, cet extrait sera un livre» ${ }^{55}$; e tale fu sentita dagli amici che in essa videro un'autentica traduzione romantica, l'unica in grado di far conoscere il vero Dante. Anche se non fu mai ripresa, la traduzione di Antoni Deschamps ebbe, nell'ambito del movimento romantico francese, una risonanza enorme, di cui è facile trovare gli echi nei giornali o nelle raccolte poetiche del tempo ${ }^{56}$.

Senza voler mettere in discussione l'originalità e l'importanza della traduzione di Antoni Deschamps, è però fuori di dubbio, e nello stesso tempo significativo, che egli, nel preparare la sua, avesse sotto gli occhi quella dell'Artaud, che, come ha fatto vedere a suo tempo Gragnier de Cassagnac, e come, sulla sua scorta, sarebbe facile far vedere anche oggi, ha ripreso in più punti in maniera quasi letterale $e^{57}$. Allo stesso modo sarebbe facile far vedere come nella scelta dei canti o dei brani da tradurre, Antoni Deschimps abbia tenuto conto, più di quanto sarebbe stato lecito aspettarsi da un romantico par suo, delle indicazioni e delle citazioni proposte a suo tempo dallo Ginguené nella sua Histoire littéraire d'Italie. Sebbene Antoni Deschamps intenda, a differenza della maggior parte di coloro che l'avevano preceduto, presentare ai suoi lettori la Commedia nel suo insieme, di fatto egli opera una scelta che privilegia, come già aveva fatto lo Ginguené, l'Inferno, di cui sono tradotti ben 11 canti, mentre il Purgatorio con sei ed il Paradiso con quattro, hanno uno spazio decisamente minore, come era accaduto per l'appunto nella presentazione che del capolavoro dantesco aveva proposto l'Histoire littéraire de l'Italie. Ancora più interessante, avendone il tempo, sarebbe esaminare i canti o gli episodi tradotti da Antoni Deschamps. Albert Counson credette di intravvedervi «les goûts du romantisme» ${ }^{58}$. Di fatto, essi coincidono, à peu de choses près, con i canti o gli episodi che lo Ginguené aveva segnalato all'attenzione dei Francesi fin dal 1811; a segno, da un lato, di una continuità che solo

(55) Cfr. La Divine Comédie de Dante Alighieri, traduite en vers français par M. ANTONI DESCHAMPS. Paris, Charles Gosselin Editeur. M.DCCC.XXX, pp. III-IV.

(56) Il «Journal des débats» del 21 febbraio 1830 elogiò, ad esempio, A. Deschamps per aver tradotto Dante ricorrendo al «nouveau système de traduction», quello messo in atto dall'école nouvelle che consiste «à rendre l'esprit et, s'il est possibile, à sauver la lettre», compito che richiede «un grand talent», come ha appunto dimostrato di avere A. Deschamps, ch'«il faut louer d'autant plus d'avoir suivi ce nouveau système, qu'il n'en est point qui convienne mieux à son poète». «Il ne [s'est pas effrayé $]$ d'importer dans notre langue des tours calqués sur ceux de Dante, et quant au ton général du poète, il [a tenu] surtout à le rendre, afin de com- muniquer au lecteur l'impression forte et singulière de l'original», faceva poi osservare l'anonimo articolista; mentre il «Mercure de France» dopo aver fatto notare che A. Deschamps aveva tradotto Dante «avec amour», precisava che «c'est ainsi qu'il faut le traduire» (t. XXVIII, p. 189). Per avere un'idea di quello che la traduzione di A. Deschamps significo per i suoi contemporanei, ed in particolare per i Romantici del Cénacle, cfr. A. Counson, Dante en France, cit., pp. 140-144 e M. Pitwood, op. cit., pp.90-97. Utili indicazioni anche in V. LAISNÉ, L'Arsenal romantique. Le salon de Charles Nodier (18241834), Paris, Champion, 2003, pp. 468-471.

(57) «Revue des deux mondes» del 15 ottobre 1830 , pp. 458 e segg.: «Revue littéraire», a firma G. C.

(58) A. Counson, Dante en France, cit., p. 141. 
la scarsa attenzione di coloro che fino a ora si sono occupati della fortuna di Dante in Francia ha impedito di vedere, dall'altro dell'importanza che l'opera dello Ginguené ha avuto nel suscitare, nei Francesi del primo Ottocento, un sempre maggiore interesse per Dante e per la sua opera.

Allo Ginguené faceva peraltro esplicito riferimento un altro degli uomini che sono all'origine dell'engouement di cui abbiamo detto pocanzi: quel Charles Fauriel, l'amico di Monti e di Manzoni, che con i suoi dotti commenti sull'Inferno dantesco alla Sorbonne nel 1832 non solo risolse molte delle oscurità che ne avevano reso difficile, fino ad allora, la lettura, ma suscitò anche l'entusiasmo di un'intera generazione di studenti, e la vocazione di non pochi giovani (Jean-Jacques Ampère e Frédéric Ozanam, tra tutti) che a Dante, negli anni successivi, avrebbero dedicato un vero e proprio culto ${ }^{59}$. A. Counson, pur ricordando le sue ascendenze voltairiane, aveva collegato Fauriel al gruppo di Coppet, quindi a Madame de Staël e agli Schlegel, ma Paul Hazard, qualche anno più tardi, ha fatto notare quanto egli dovesse invece, nella formazione, nel metodo e nel gusto, allo Ginguené, di cui possedeva le principali caratteristiche critiche: esigenza di una conoscenza approfondita dell'argomento studiato, ostilità nei riguardi dei pregiudizi e delle idées reçues, volontà di capire l'altro alla luce della sua storia e delle sue peculiarità, convinzione che ogni paese abbia il diritto di esprimere il bello nelle forme che le sono proprie, rifiuto di scambiare i propri «préjugés par des principes» ${ }^{60}$.

Un discorso non molto diverso potrebbe farsi per Abel Villemain, l'altro grande professore della Sorbonne che, in quegli stessi anni, contribuì a far amare Dante a tutta una generazione di giovani, e che pure non sarebbe difficile collegare ad un filone di studiosi che ha in Sismondi, in Guizot e, ancora una volta, in Ginguené i suoi rappresentanti più significativi. Quello che abbiamo detto fin qui dimostra tuttavia, ci pare, a sufficienza l'assunto dal quale siamo partiti: vale a dire che se fu indubbiamente nel decennio 1830-1840 che l'engouement per Dante raggiunse in Francia il suo apice, fino a trasformarsi in una vera e propria moda, l'interesse per Dante e per la sua opera nacque, di fatto, nei decenni precedenti, grazie a studiosi o uomini di cui la storia ha magari conservato meno il ricordo, ma che con la loro opera e con il loro amore per Dante ed il suo capolavoro hanno saputo preparare il terreno e creare le condizioni affinché la generazione romantica potesse fare di Dante uno dei suoi idoli o delle sue icone: da ammirare e da sbandierare magari più di quanto non l'abbia amato e capito fino in fondo, come una lettura attenta dell'influenza esercitata da Dante sui romantici francesi lascerebbe abbastanza facilmente vedere (dando così ulteriore ragione alla tesi di Jacqueline Risset ricordata all'inizio di questo intervento). Ma questo è un altro discorso, che meriterebbe ben altro tempo, ed un'analisi assai più dettagliata ${ }^{61}$.

FRANCO PIVA

(59) Nello studio che abbiamo ricordato sopra Sainte-Beuve parlava, al di là delle differenze tra $\mathrm{i}$ discepoli, di una vera e propria «école de Fauriel» (p. 73).

(60) P. HazARD, La Révolution française et les lettres italiennes, Paris, Hachette, 1910, p. 462.

(61) Utili, a questo proposito, le conclusioni alle quali è pervenuto, alla fine della sua indagine, M. PITWOOD (op. cit., pp. 265-274); ma molto utili anche le considerazioni formulate, al riguardo, da Lionello Sozzi in alcuni suoi recenti interventi (Dante e Hugo, «Letture classensi», n. 14 [1985], pp. 45-61 e, soprattutto, Dante in Francia dai Romantici a Baudelaire, «Letture classensi», n. 19 [1990], pp. 23-33). 